\title{
A New Non-Surgical Alternative to Manipulations Under Anesthesia Following Knee Replacement
}

\author{
D Carl Freeman ${ }^{1 *}$ and Robert J Ference ${ }^{2}$ \\ ${ }^{1}$ Department of Biological Sciences, Wayne State University, USA \\ ${ }^{2}$ DMC Harper University Hospital, USA
}

*Corresponding author: D. Carl Freeman, Department of Biological Sciences, Wayne State University, Detroit MI 48202, USA.

\begin{abstract}
Stiff knee occurs in between $2 \%$ and $23 \%$ of total knee arthroplasty patients. Resolution of a stiff knee is critical for patients' quality of life following a TKA. Historically, treatment options include physical therapy, manipulation under anesthesia, arthroscopic lysis, and open lysis. Excluding physical therapy, the other options all require anesthesia, with procedures usually being done in a hospital or surgical center. Here, we report on a new non-surgical alternative that is done in patients' homes and is as efficacious as a manipulation under anesthesia.
\end{abstract}

Keywords: Stiff knee; Manipulation under anesthesia; X10; Total knee arthroplasty; Flexion

\section{Introduction}

Arthrofibrosis of the knee, stiff knee, is a common complication of knee surgery, with an incidence rate of $2 \%$ to $23 \%$ [1]. Stiff knee is usually defined as an inability to flex the knee to $90^{\circ}$ [2]. Risk factors for stiff knee include being African American, female, less than 60 years old, nicotine use [3] and multiple surgeries [4]. LaHaise et al. [5] report that discharge range of motion (ROM) is a reliable predictor of stiff knee. However, they also report that BMI is negatively related to the frequency of stiff knee. Whereas Gadinsk et al. [6] report that BMI is strongly positively related to the frequency of stiff knee. These conflicting results suggest there is the possibility of confounding factors involved and that very large sample sizes will be needed to resolve the issue. Recently, Arshi et al. [7] report that patients undergoing outpatient total knee arthroplasty are $28 \%$ more likely to have a stiff knee than patients undergoing inpatient knee arthroplasty. Interestingly, Pamilo et al. [8] found that fast-tracked TKA patients did not have an increase in stiff knee (manipulation under anesthesia) rates compared to inpatient total knee replacements.

Treatment options available for treating stiff knee, range from physical therapy, manipulation under anesthesia (MUA), arthroscopic lysis, to open lysis, with MUA having the largest gains in ROM [2], especially when performed early and open lysis having the least gain in ROM [2]. MUA is the most common treatment for stiff knee [2,3] and is usually recommended if patients have not achieved $90^{\circ}$ of flexion, though the time span for making the assessment ranges from two weeks to over three months postsurgery [2]. Typically, MUAs resulted in patients having $101^{\circ}$ of flexion, if performed after six weeks post-surgery [1,4] However Newman et al.4 found that for MUAs performed before six weeks the patients achieved $106.7^{\circ}$ ROM. Nevertheless, the average of ROMs failed to reach the $110^{\circ}$ of flexion required to perform most activities of daily living [9]. Desai et al. [10], found that $24 \%$ of their patients required a second MUA, unfortunately these second MUAs did not lead to gains in ROM.

Our purpose here is to introduce the new non-surgical, low pain alternative to MUAs, the in home X10 knee rehabilitation machine (Figure 1). This computer-controlled device has a robotic arm that is moved by pressure to incrementally increase the range of motion. Once the patient has established their maximum pressure threshold, the machine will use that amount of force to move the patient's leg (controllable to within one pound of pressure and one degree of flexion). However, as the session progresses the pressure needed to reach a given degree of flexion declines, enabling the patient to increase their flexion and then gradually increase the 
pressure pushing their leg, to achieve even more flexion. The X10's ultrasensitive pressure sensors sense when a patient has reached their preset pressure level and the machine will stop and reverse to protect the patient from pain. Because the machine is used in home and causes little therapeutic pain, patients are encouraged to use it three or more times a day, seven days a week. Since the machine is gentle, the compliance is high (>92\%). Below we retrospectively examine the data of 17 patients who had stiff knees and subsequently used the X10, without an MUA, to regain their flexion. All patients had had a TKA followed by standard physical therapy which had failed to yield the required $90^{\circ}$ of flexion (Figure 1).

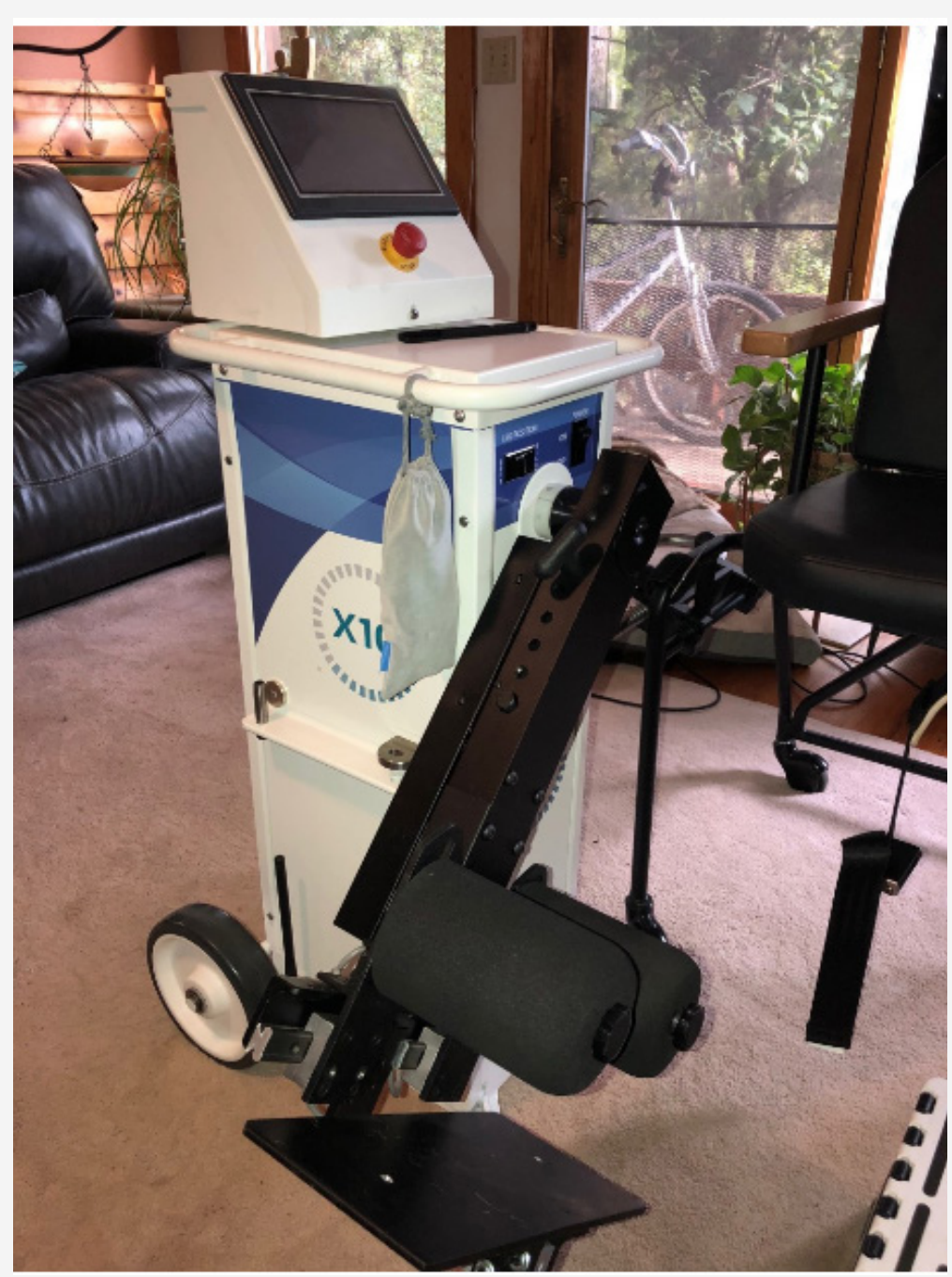

Figure 1: Patient on the $\mathrm{X} 10$

\section{Methods}

Patient age, gender, BMI, their initial flexion and extension, and their final extension and final flexion were all assessed. Nondemographic data were obtained from the X10 machine which transmits the flexion and extension of each stroke the patients make to a HIPPA compliant server. The data were then downloaded to a spreadsheet for analysis. The data are accurate to within one degree of arc. As dependent variables, we assessed the final flexion, the number of days post-surgery, the number of days the patient used the X10, and the gain in flexion.

\section{Result}

The mean age of patients was 62.3 years (range 49-76), 35.3\% male, mean BMI was 30.7, (range 20-42). The average patient began using the X10 116.1 days post-surgery (range 49-675). There was a trend for increasing initial flexion as the days post-surgery increased $(\mathrm{R}=0.361, \mathrm{P}<0.159)$. The average patient had an initial flexion of $82.8^{\circ}$, (range $50^{\circ}-110^{\circ}$ ). The average patient used the machine for 23.4 days (range 10-47). The average patient achieved $116.9^{\circ}$ of flexion (range $90^{\circ}-130^{\circ}$ ) with an average gain of $27.9^{\circ}$ (range $15^{\circ}$ $50^{\circ}$ ). All patients achieved at least $90^{\circ}$ of flexion and over $82 \%$ of patients achieved greater than $110^{\circ}$ of flexion indicating that they could engage in activities of daily living [9].

\section{Discussion}

The $\mathrm{X} 10$ is comparable to the outcomes of the surgical procedures for treating knee arthrofibrosis [2]. The X10 has advantages over the surgical techniques: in that it is not painful, does not require anesthesia or additional physical therapy after its use, and is less expensive than the surgical procedures. While the 
surgical procedures accomplish their goal in a one-time event and hence the need for anesthesia, the X10 does require a few weeks of use, but then accomplishes the follow up physical therapy at the same time.

\section{Conclusion}

The new X10 patented technology provides an increase in flexion after standard physical therapy has failed. The X10 is not surgical, does not require anesthesia, is not painful, and does not require additional physical therapy upon completion. Accordingly, we recommend that the X10 be the first response to stiff knees.

\section{Acknowledgement}

We wish to thank Halley Orthopedics for their data, and Aaron Laske, Lisa Pelley, and Erin Rempher for their help in the preparation of the data.

\section{Conflict of Interest}

No conflict of interest.

\section{References}

1. Choi H-R, Siliski J, Malchau H, Freiberg A, Rubash H, et al. (2014) How often is functional range of motion obtained by manipulation for stiff total knee arthroplasty? Int Orthop 38(08): 1641-1645.

2. Fitzsimmons SE, Vazquez EA, Bronson MJ (2010) How to treat the stiff total knee arthroplasty? A systematic review. Clin Orthop Relat Res 468(4): 1096.
3. Pfefferle KJ, Shemory ST, Dilisio MF, Fening SD, Ian M Gradisar (2014) Risk Factors for Manipulation After Total Knee Arthroplasty: A Pooled Electronic Health Record Database Study. The Journal of Arthroplasty 29(10): 2036-2038.

4. Newman, ET, Herschmiller, TA, Attarian, DE, Vail TP, Bolognesi MP, et al. (2018) Risk Factors, Outcomes, and Timing of Manipulation Under Anesthesia. After Total Knee Arthroplasty. J of Arthroplasty 33(1): 245249.

5. La Haise KM, Vargo DV, Barrazueta GA, Nairus JG, Bono JV, et al. (2019) Range of motion at discharge predicts need for manipulation following total knee arthroplasty. J Knee Surgery.

6. Gadinsky NE, Ehrhardt JK, Urband C, Westrich GH (2011) Effect of body mass index on range of motion and manipulation after total knee arthroplasty. Journal of Arthroplasty 26(8): 1194-1197.

7. Leong NL, D Oro A, Wang C, Buser, Z, Zang, JC, et al. (2017) Outpatient Total Knee Arthroplasty Is Associated with Higher Risk of Perioperative Complications. The Journal of Bone and Joint Surgery 99(23): 19781986.

8. Pamilo KJ, Torkki P, Peltola M, Pesola M, Remes, V et al. (2018) Fasttracking for total knee replacement reduces use of institutional care without compromising quality. A register-based analysis of 4 hospitals and 4,256 replacements. Acta Orthopaedica 89(2): 184-189.

9. Rowe PJ, Myles CM, Nutton R, (2005) The effect of total knee arthroplasty on joint movement during functional activities and joint range of motion with particular regard to higher flexion users. Journal of Orthopaedic Surgery Hong Kong 13(2): 131-138.

10. Desai AS, Karmegam A, Dramis A, Board TN, Raut, V (2014) Manipulation for stiffness following total knee arthroplasty: when and how often to do it? Eur J Orthop Surg Traumatol 24(7): 1291-1295. 